ARTICLE

\title{
Unravelling strong electronic interlayer and intralayer correlations in a transition metal dichalcogenide
}

\author{
T. J. Whitcher ${ }^{1,2,3 凶}$, Angga Dito Fauzi (10 1,2, D. Caozheng (D) ${ }^{2}$, X. Chi ${ }^{2,3}$, A. Syahroni $^{4}$, T. C. Asmara (1) ${ }^{1}$,

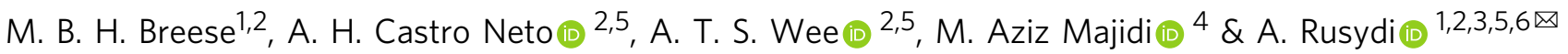

Electronic correlations play important roles in driving exotic phenomena in condensed matter physics. They determine low-energy properties through high-energy bands well-beyond optics. Great effort has been made to understand low-energy excitations such as low-energy excitons in transition metal dichalcogenides (TMDCs), however their high-energy bands and interlayer correlation remain mysteries. Herewith, by measuring temperature- and polarization-dependent complex dielectric and loss functions of bulk molybdenum disulphide from near-infrared to soft X-ray, supported with theoretical calculations, we discover unconventional soft X-ray correlated-plasmons with low-loss, and electronic transitions that reduce dimensionality and increase correlations, accompanied with significantly modified low-energy excitons. At room temperature, interlayer electronic correlations, together with the intralayer correlations in the $c$-axis, are surprisingly strong, yielding a three-dimensionallike system. Upon cooling, wide-range spectral-weight transfer occurs across a few tens of eV and in-plane $p$ - $d$ hybridizations become enhanced, revealing strong Coulomb correlations and electronic anisotropy, yielding a two-dimensional-like system. Our result shows the importance of strong electronic, interlayer and intralayer correlations in determining electronic structure and opens up applications of utilizing TMDCs on plasmonic nanolithrography.

\footnotetext{
${ }^{1}$ Advanced Research Initiative for Correlated-Electron Systems (ARiCES), Department of Physics, National University of Singapore, 2 Science Drive 3 , Singapore 117576, Singapore. ${ }^{2}$ Singapore Synchrotron Light Source, National University of Singapore, 5 Research Link, Singapore 117603 , Singapore. ${ }^{3}$ Centre for Advanced 2D Materials, National University of Singapore, 2 Science Drive 3, Singapore 117546, Singapore. ${ }^{4}$ Department of Physics, University of Indonesia, Depok 16424, Indonesia. ${ }^{5}$ NUSSNI-NanoCore, National University of Singapore, Singapore 117576, Singapore. ${ }^{6}$ NUS Graduate School for

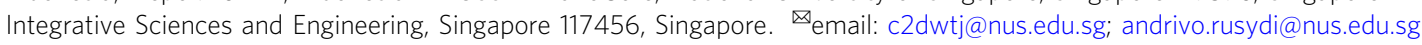


E lectronic correlations have played an important role in generating many rich and exotic phase diagrams in strongly correlated electron systems ${ }^{1-3}$. These include colossal magnetoresistance $(\mathrm{CMR})^{4,5}$, metal-insulator transitions ${ }^{6,7}$, and high-energy resonant excitons ${ }^{8,9}$, that are manifested through many-body interactions. Recently, strong electronic correlation effects have also been found to drive plasmons that are found in topological insulators such as bismuth selenide $\left(\mathrm{Bi}_{2} \mathrm{Se}_{3}\right)$ when they are cooled ${ }^{10,11}$. This is due to the suppression of the electronic screening, which enhances long-range and high-energy electron-electron correlations, revealing topological insulators as strongly-correlated materials.

An important implication of electronic correlations is that high-energy bands, which are well beyond the optical regime, are tightly connected with low-energy bands, with the result being that low-energy properties, such as low-energy excitons, transports, or magnetism, may be determined by high-energy bands. These strong correlations can directly be probed. They show a unique spectral weight transfer, as a function of temperature or doping, which is a fingerprint of the complex dielectric function and loss function of a material, and involve high-energy and lowenergy bands simultaneously fulfilling the charge conservation and $f$-sum rule ${ }^{1,12-15}$.

A group-VI low-dimensional transition metal dichalcogenide (TMDC) such as molybdenum disulphide $\left(\mathrm{MoS}_{2}\right)$ is of particular interest because of its rich fundamental properties and potential applications. Recently, low-energy electronic properties of monolayer $\mathrm{MoS}_{2}$, have been heavily investigated due to its transformation from an indirect band-gap semiconducting material in its bulk form to a direct band-gap semiconductor ${ }^{16-18}$. Most studies have been focused on the formation of low-energy excitons and the effects of lowdimensional confinement ${ }^{19-21}$, which were shown to give rise to photoluminescence in the monolayers ${ }^{22,23}$, and the development of valleytronics based on the spin-orbit splitting of the valence and conduction bands near the band-gap ${ }^{24,25}$. It has been observed that the low-energy excitons have changed as a function of temperature, however it requires high-energy bands to understand the low-energy excitations. Since there has been no direct experimental techniques to simultaneously probe high-energy and low-energy bands and how high-energy bands affect low-energy properties, origins of low-energy properties remain hotly debated.

Whilst the electronic properties of bulk-like, layered $\mathrm{MoS}_{2}$ were investigated several decades ago ${ }^{26-28}$, the properties of bulk-like, layered materials, such as $\mathrm{MoS}_{2}$, in comparison to monolayers, are far from understood and shall not be dismissed. In particular, bulk-like materials are necessary to understand quantum confinement effects, and an electronic transition to a lowdimensionality due to an interplay of interlayer and intralayer electronic correlations could have significant effects. Indeed, further studies have shown that such low-energy properties dramatically change with thickness, from a monolayer toward a bulk-like, layered material, such as the enhancement of spinpolarisation from single-layer to bulk $\mathrm{WS}_{2}{ }^{29}$, the screening of Coulomb correlations from two-dimensional graphene to bulklike, layered graphite ${ }^{30}$, and the recent discovery of unconventional superconductivity in graphene superlattices ${ }^{31}$. This raises the fundamental question on the role of electronic, interlayer and intralayer correlations in two-dimensional systems. It is therefore crucial to measure the temperature-dependent and polarizationdependent complex dielectric and loss functions of such bulk-like, layered systems at energies beyond the optical regime.

In all previous studies, the optical properties of TMDCs, particularly for above $3-4 \mathrm{eV}$, were mainly measured using reflectance or energy loss spectroscopies or a combination of these techniques ${ }^{28,32-34}$. While these techniques are useful, they have fundamental limitations, which lead to inaccurate optical spectra for the following reasons. First, in order to obtain the complex dielectric function and loss function from reflectance and energy loss spectroscopy measurements, one has to perform Kramers-Kronig transformations (KKT), which requires a much broader energy range than the measurement capabilities in order to achieve a stable $\mathrm{KKT}^{35}$. However, all existing optical measurements on TMDCs were performed in a limited energy range, therefore the data analysis required extrapolative assumptions of spectra for energies beyond the measured spectral range, which may result in inaccurate low-energy optical spectra. One of the fundamental characteristics resulting from KKT is that any change, even relatively small ones, in the high-energy spectrum would significantly influence the low-energy spectrum $^{36-38}$. Therefore, the limitations on energy measurements and theoretical assumptions made for the high-energy spectra that were temperature-independent and doping-independent, result in unstable KKT, which yields inaccurate complex dielectric functions and loss functions ${ }^{35-37}$. Second, while optical reflectivity, which is a photon-in photon out technique, is bulk sensitive, electron loss spectroscopies are very surface sensitive. Therefore, the normalization and combination of spectra with different penetration depths lead to another discrepancy. Third, since most bulk TMDC systems are actually insulating, electron-loss spectroscopies face so-called charging problems, which then lead to inaccurate spectra and significant broadening and shifting of peaks. Therefore, there was no reliable experimental data of highenergy bands on TMDCs.

In this article, we address this fundamental problem and surprisingly discover unconventional, many-body soft X-ray correlated-plasmons with low-loss in bulk, single crystal $\mathrm{MoS}_{2}$ using a new methodology. Interestingly, upon cooling from 400 to $40 \mathrm{~K}$, we find two electronic transitions; a sharp transition from threedimensional-like to two-dimensional-like system at $\sim 150 \mathrm{~K}$, and a further enhancement of strong electronic correlations at $\sim 100 \mathrm{~K}$, accompanied with significantly modified low-energy excitons. At higher temperatures from $400 \mathrm{~K}$ down to $150 \mathrm{~K}$, a soft X-ray correlated-plasmon with low-loss and three-dimensional character is observed at $\sim 35 \mathrm{eV}$. Surprisingly, Mo $p-\mathrm{S} d$ hybridizations are isotropic between the in-plane and out-of-plane directions, revealing strong interlayer and intralayer correlations yielding a three-dimensional-like system. Upon cooling below $150 \mathrm{~K}$, inplane (out-of-plane) Mo $p-S d$ hybridizations become enhanced (reduced), revealing a two-dimensional-like system within the bulk-like system. Further cooling below $100 \mathrm{~K}$, anomalous spectral-weight transfer occurs from high-energies $(>3.4 \mathrm{eV})$ to low-energies bands $(\leq 3.4 \mathrm{eV})$ revealing strong electronic correlations. As a result of the interplay between strong electronic, interlayer and intralayer correlations, the high-energy bands play a crucial role in determining low-energy properties such as excitons, which become dramatically enhanced and sharpened and their peak energies are blue-shifted.

These direct observations are made using a newly developed experimental technique at the Soft X-ray-Ultraviolet (SUV) beamline of the Singapore Synchrotron Light Source that is capable of combining and analyze Mueller-Matrix spectroscopic ellipsometry, soft X-ray reflectivity, and X-ray absorption spectroscopy measurements as a function of temperature and polarizations in a broad energy range, from 0.6 to $1500 \mathrm{eV}$. The Mueller-Matrix spectroscopic ellipsometry, which is a selfnormalized technique, directly probes the complex dielectric function, loss function, and reflectivity simultaneously in the energy range from 0.6 to $5.5 \mathrm{eV}$ without the need for $\mathrm{KKT}^{37,38}$, while the soft X-ray reflectivity covers the energy range from 3.5 to $1500 \mathrm{eV}^{39}$. Since both techniques are photon-in photon-out 

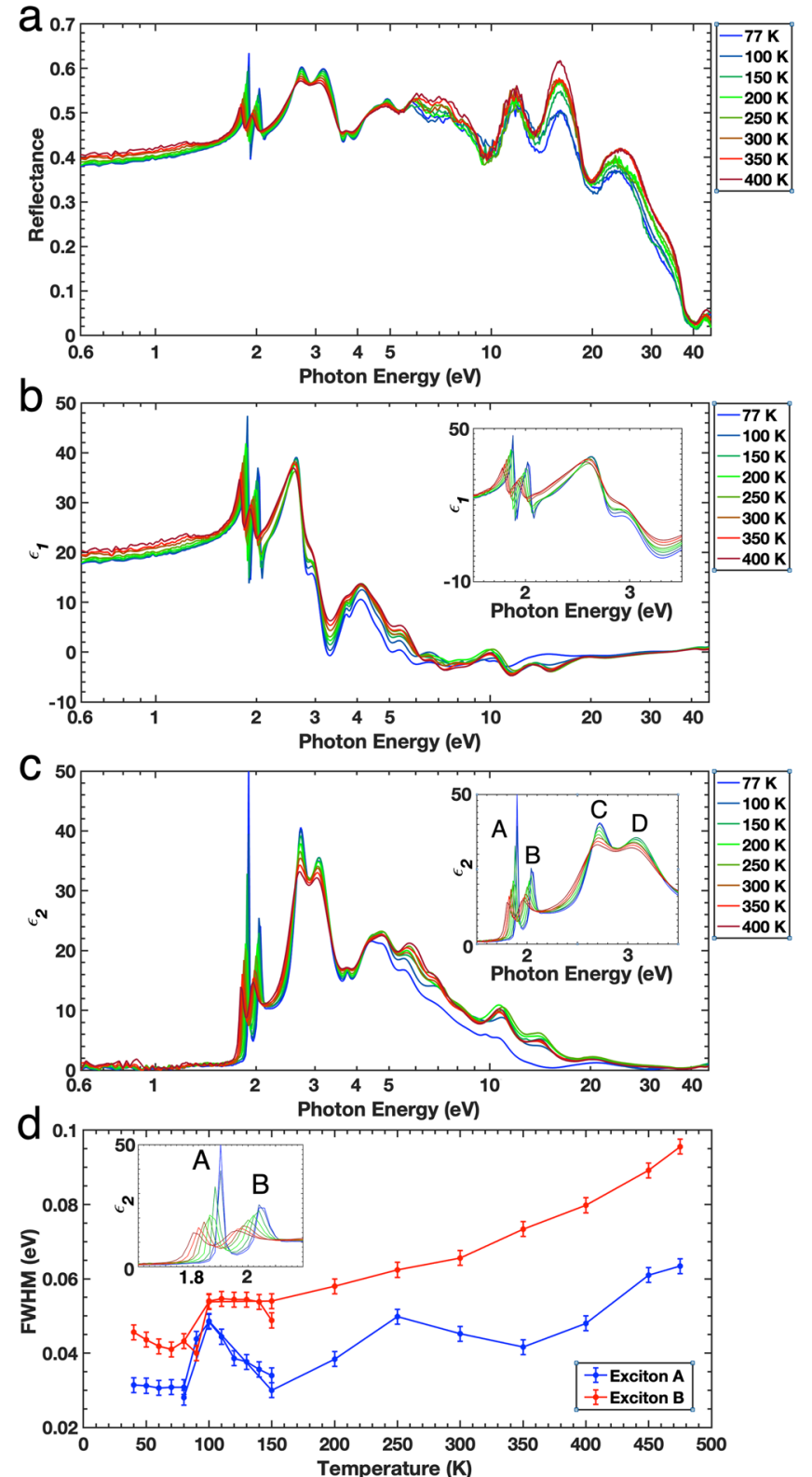

Fig. 1 Reflectance measurements and complex dielectric function of $\mathrm{MoS}_{2}$ as a function of temperature. a Measurements of the reflectance of the bulk $\mathrm{MoS}_{2}$ from the near-infrared $(0.6 \mathrm{eV})$ up to soft $\mathrm{X}$-ray $(45 \mathrm{eV})$ using the SUV beamline at SSLS, log scale. The $\mathbf{b}$ real $\left(\varepsilon_{1}\right)$ part and $\mathbf{c}$ imaginary $\left(\varepsilon_{2}\right)$ part of the complex dielectric function of $\mathrm{MoS}_{2}$ from spectroscopic ellipsometry and soft $\mathrm{X}$-ray reflectance, log scale. $\mathbf{d}$ The FWHM of excitons $A$ and $B$ as a function of temperature showing significant change. Error bars are calculated using a $2 \mathrm{meV}$ shift. Insets: Magnified view of complex dielectric function of low-energy excitons from $\mathbf{b}$ and $\mathbf{c}$ on a linear scale.

measurements and can cover an energy range from the infrared to the soft X-ray with high-energy resolution, the combination of these techniques leads to a stable KKT.

\section{Results and discussion}

Figure 1 shows reflectance measurements and the complex dielectric function of $\mathrm{MoS}_{2}$, measured at selected photon energy ranges from 0.6 to $45.0 \mathrm{eV}$ and as a function of temperature, taken using a combination of the Soft X-ray reflectivity and Mueller-Matrix spectroscopic ellipsometry (see Supplementary Figs. 1 and 2 for details). We also implement a stabilized normalization procedure in which the low energy spectra of the Soft X-ray reflectivity measurements are self-normalizing via spectroscopic ellipsometry and the high-energy spectra are normalized using the low energy spectra and the tabulated values at the high-energy end of the spectrum, yielding a stabilized KKT across all temperatures ${ }^{37,40}$. Utilising this method also shows the significant impact that higher energy measurements have on the determination of the complex dielectric function at lower energies (See Supplementary Fig. 3) and that the complex dielectric function is significantly underestimated near the limits of spectral measurements.

Figure 1a shows surprisingly strong temperature-dependent $\mathrm{MoS}_{2}$ reflectivity in a broad energy range, which directly reveals strong electronic correlations in $\mathrm{MoS}_{2}$. The real $\varepsilon_{1}$ (Fig. 1b) and imaginary $\varepsilon_{2}$ (Fig. 1c) parts of the complex dielectric function of $\mathrm{MoS}_{2}$ show a strong temperature dependence. For further clarity, Supplementary Fig. 2a shows the linear plot of the reflectance and Supplementary Figs. $2 \mathrm{~b}$ and $1 \mathrm{c}$ show the linear plot of the complex dielectric function in order to highlight all the features over the wide energy range. The refractive index, $n$, and the absorption coefficient, $k$, are also shown as a function of temperature in Supplementary Fig. 4.

Below $3.4 \mathrm{eV}, \varepsilon_{1}$ and $\varepsilon_{2}$ show the presence of the four excitonic features that are commonly labelled $A-D^{21}$ (see inset of Fig. 1b, c, respectively). We find two electronic transitions, at $\sim 150$ and $\sim 100 \mathrm{~K}$. As the temperature decreases from 475 to $40 \mathrm{~K}$, the intensity of the low-energy exciton peaks, A and B, become enhanced and their peak energies are blue-shifted (see also Supplementary Fig. 5 for details). Interestingly, the full-width at halfmaximum (FWHM) of excitons $\mathrm{A}$ and $\mathrm{B}$ sharpens significantly by over a factor of 2 , from $\sim 60$ to $\sim 30 \mathrm{meV}$ and $\sim 95$ to $\sim 45 \mathrm{meV}$, respectively (Fig. 1d). For reproducibility and details, we perform the experiments twice, i.e., the broad range of temperatures initially measured and the detailed temperature measurements that followed. There is a clear difference in the effect of temperature on the FWHM for both excitons with the electronic transitions observed at $\sim 150$ and $100 \mathrm{~K}$. As the $\mathrm{MoS}_{2}$ is cooled from 400 to $150 \mathrm{~K}$, the FWHM of excitonic peak A decreases, with some fluctuations suggesting the existence of competing interactions. Further cooling from 150 to $100 \mathrm{~K}$, results in the FWHM broadening significantly and then from 100 to $40 \mathrm{~K}$, it narrows sharply again. A similar, less intense event also occurs around $350 \mathrm{~K}$ due to the slight increase in the FWHM of exciton A from 350 to $250 \mathrm{~K}$ before a decrease down to $150 \mathrm{~K}$. The FWHM of excitonic peak B steadily decreases from 400 to $150 \mathrm{~K}$, and remains nearly temperature independent between $150 \mathrm{~K}$ and $100 \mathrm{~K}$, before it suddenly sharpens below $100 \mathrm{~K}$. The FWHM of the excitons are directly linked to their binding energies and lifetimes, thus there must be some kind of energetic event that occurs, particularly below 350 and $150 \mathrm{~K}$, that causes the lowenergy excitons to become unstable (broadening indicates reduced life-times and lowered binding energies ${ }^{35,41}$ ) and then to re-stabilise rapidly below $100 \mathrm{~K}$. As discussed later, it is found that the temperature dependent low-energy excitons are determined not only by the spectral weight of higher-energy bands, but also the dimensionalities of the material, i.e., whether they are electronically two-dimensional or three-dimensional, particularly the unconventional soft X-ray correlated-plasmons.

An important aspect highlighted by the extended complex dielectric function is the various changes in spectral weight seen across the spectral region. For high-energies above $3.4 \mathrm{eV}$, interestingly, we observe four significant peaks at higher energies of $\sim 5, \sim 6, \sim 11$, and $\sim 15 \mathrm{eV}$ that are very strongly temperaturedependent (Fig. 1). The most startling result is drastic changes of the complex dielectric function above $13 \mathrm{eV}$ with two transition temperatures, $\sim 150$ and $\sim 100 \mathrm{~K}$. In contrast, below $3.4 \mathrm{eV}$, the 

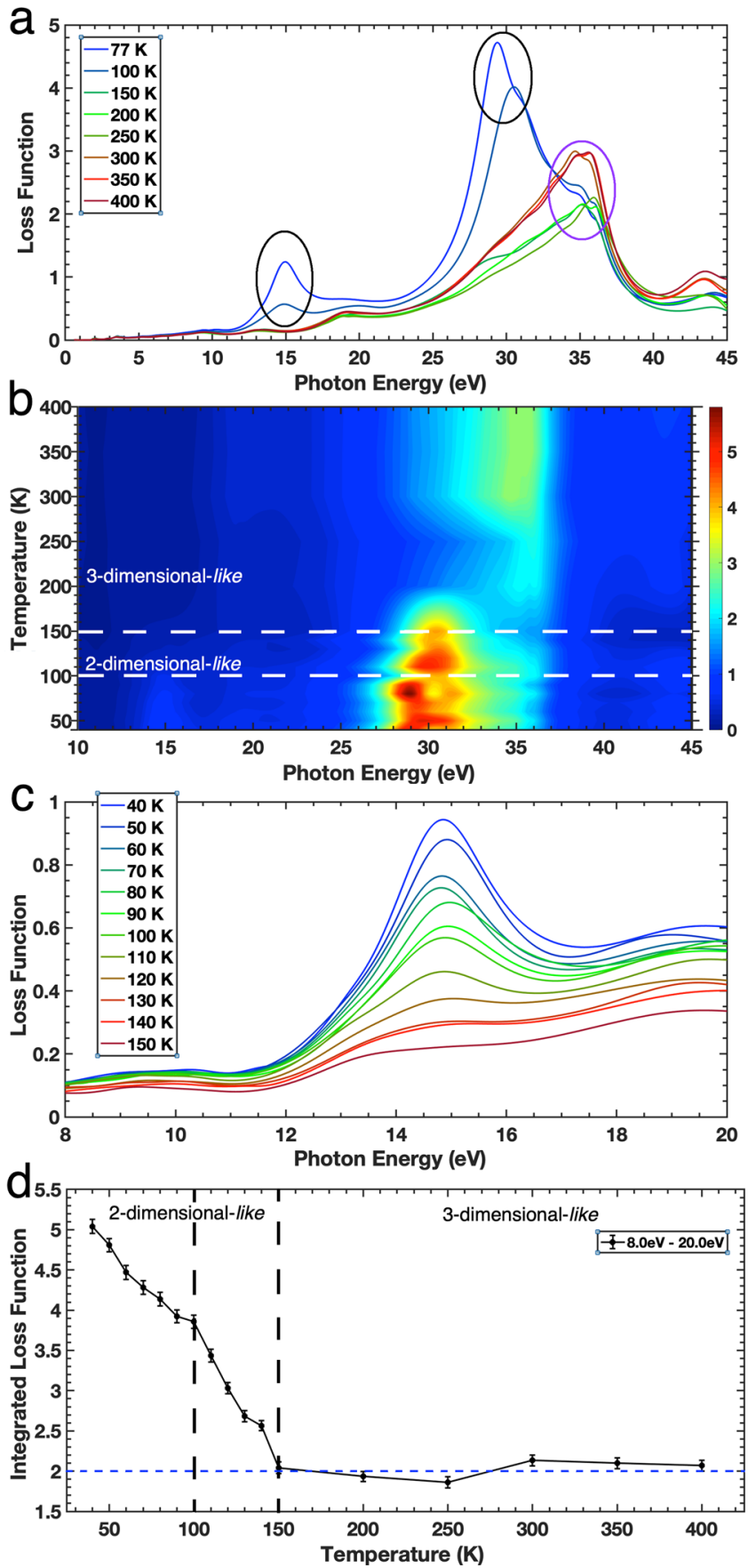

Fig. 2 Loss function and spectral weight of bulk $\mathbf{M o S}_{\mathbf{2}}$. a The loss function of $\mathrm{MoS}_{2}$ as a function of temperature from 0.6 to $45 \mathrm{eV}$. The soft X-ray correlated-plasmonic peaks are circled, i.e., black and purple circles are for low-temperature 2-dimensional-like and high-temperature 3-dimensionallike soft X-ray correlated-plasmons, respectively. b Contour plot of loss function shows transitions at 150 and $100 \mathrm{~K}$. Note that vertical dashed lines represent the transition temperatures. c Detailed measurements of the loss function of $\mathrm{MoS}_{2}$ from 8 to $20 \mathrm{eV}$ in the temperature range of $40-150 \mathrm{~K}$ at $10 \mathrm{~K}$ intervals. $\mathbf{d}$ Evolution of the soft $\mathrm{X}$-ray correlated-plasmon at $\sim 15 \mathrm{eV}$ as a function of temperature. Note that the dashed vertical lines represent the transition temperatures and dotted horizontal line is a background level. Error bars are calculated using a $20 \mathrm{meV}$ shift.

lower temperatures dominate the amplitude, whereas the higher temperatures show much broader peaks (See Supplementary Fig. 3). Above $8 \mathrm{eV}$ the mid-range temperatures of 200 and $250 \mathrm{~K}$ dominate the spectral weight whilst the hotter temperatures lose spectral weight.

Our main observation is unconventional soft X-ray correlatedplasmons, one at $\sim 35 \mathrm{eV}$ observed even from high temperature of well above room temperature and a pair at $\sim 15$ and $\sim 30 \mathrm{eV}$ observed below $\sim 150 \mathrm{~K}$ as seen in the loss function of $\mathrm{MoS}_{2}$ shown in Fig. 2 (highlighted in Fig. 2a, b). The loss function is directly calculated from the complex dielectric function by

$$
\mathrm{LF}=-\operatorname{Im}(1 / \varepsilon)=-\operatorname{Im}\left(1 /\left(\varepsilon_{1}+i \varepsilon_{2}\right)\right),
$$

across the entire spectral region for all the temperatures measured. It is very sensitive for any plasmonic activity within the material. For photons of energy $<1 \mathrm{keV}$, the momentum transfer, $q$, is finite but approaches zero because it is much less than the crystal momentum. In this limit, the distinction between the longitudinal and transverse $\varepsilon(\omega)$ vanishes i.e.,

$$
\lim _{q \rightarrow 0} \varepsilon_{l}(q, \omega)=\varepsilon_{t}(q, \omega),
$$

which allows spectroscopic ellipsometry and soft X-ray reflectivity measurements to probe both the optical and plasmonic properties in the low- $q$ limit ${ }^{8,41}$.

It is clear that the loss function of $\mathrm{MoS}_{2}$ at the lower temperatures of 150 and $100 \mathrm{~K}$ is significantly different from that at higher temperatures. At $400 \mathrm{~K}$, we observe a loss function peak at $\sim 35 \mathrm{eV}$ that persists down to $40 \mathrm{~K}$. The soft X-ray peak at $\sim 35 \mathrm{eV}$ in the loss function shows unique characteristics of correlatedplasmons $^{11}$, in which the peak only occurs in the loss function and is not observable in $\varepsilon_{2}$ (Fig. 1c), while $\varepsilon_{1}$ is nearly zero but remains positive (Fig. 1b), and the reflectivity show a local minimum (Fig. 1a). These properties are shown more clearly in Supplementary Fig. 4. This reveals that the $\sim 35 \mathrm{eV}$ peak is a new unconventional plasmon in the soft $\mathrm{X}$-ray regime of the strongly correlated-material $\mathrm{MoS}_{2}$, namely a soft X-ray correlated-plasmon. Having a positive $\varepsilon_{1}$ reveals very weak screening, therefore the Coulomb correlations are strong. The loss function remains temperature independent upon cooling from 400 to $300 \mathrm{~K}$. The intensity of the $\sim 35 \mathrm{eV}$ soft X-ray correlated-plasmon reduces as $\mathrm{MoS}_{2}$ is cooled to $150 \mathrm{~K}$. This is unusual as, typically, plasmons should become stronger at lower temperatures.

Interestingly, upon lowering the temperature below $150 \mathrm{~K}$, a pair of soft X-ray plasmons occurs at $\sim 15$ and $\sim 30 \mathrm{eV}$ and coherently gets stronger upon cooling. Since these $\sim 15$ and $\sim 30 \mathrm{eV}$ peaks only occur in the loss function and are not observable in $\varepsilon_{2}$ (Fig. 1c) and $\varepsilon_{1}$ is nearly zero but still positive (Fig. 1b), these are a pair of soft X-ray correlated-plasmons at low temperature and the Coulomb correlations are getting stronger upon cooling. The soft X-ray correlated-plasmons are close to the density of states where the S $3 s$ hybridizes with the Mo $4 d$ states lying between the valence bands and the core levels (namely semicore states) $27,28,42,43$. Furthermore, previous theoretical calculations of the energy electron loss function have suggested strong plasmonic character at this energy 44 . Subsequently, hybridizations in the semi-core play a role in generating the soft X-ray correlated-plasmons.

In Fig. 2c, we show the temperature dependent soft X-ray correlated-plasmon at $\sim 15 \mathrm{eV}$ because its structure is less complex and low loss as $\varepsilon_{2}$ is nearly zero. Details measurements of the complex dielectric function between 0.6 and $45 \mathrm{eV}$ within the temperature range $40-150 \mathrm{~K}$ at $10 \mathrm{~K}$ intervals are then undertaken to measure the rise of the cold soft X-ray correlated-plasmon. As the temperature decreases below $150 \mathrm{~K}$, the soft X-ray plasmon shows up and its intensity start to increase steadily with another transition at $\sim 100 \mathrm{~K}$ (Fig. $2 \mathrm{~d}$ ). The appearance of the soft $\mathrm{X}$-ray correlated-plasmons clearly has significant effects on the 
electronic structures of the material and this also nicely explains the destabilisation of the low-energy excitons seen in Fig. 1d with different transition temperatures. It is revealed that the formation of the soft X-ray correlated-plasmons has a direct impact in stabilising the whole system. However, they are not a result of any structural changes as the crystal structure of $\mathrm{MoS}_{2}$ is stable from $773 \mathrm{~K}$ down to $10 \mathrm{~K}$ and no structural transitions occur ${ }^{45,46}$. In order to shed some light on the existence of these soft X-ray correlated-plasmons and why they decrease and increase in intensities as the temperature is lowered, we examine the spectral weight transfer and hybridizations through temperaturedependent and polarization-dependent soft X-ray absorption spectroscopy, and extended band-structure and complex dielectric function calculations.

Figure $3 \mathrm{a}$ shows the optical conductivity of the $\mathrm{MoS}_{2}$ for a selected number of temperatures. By analysing the transfer of the spectral weight of the optical conductivity, $\sigma_{1}$ between 0.6 and $45 \mathrm{eV}$ as a function of temperature, we determine the origin of these high-energy plasmons at low temperatures. For this analysis, the measured spectral range is divided into three spectral regions as follows; I: $0.6-3.4 \mathrm{eV}$, II: $3.4-9.0 \mathrm{eV}$ and III: 9.0-45.0 eV, with error bars are calculated based on $20 \mathrm{meV}$ shift on either side of spectral divide.

The integration of the optical conductivity is related to the freecharge carrier density, $n_{\mathrm{e}}$, via the charge conserving $f$-sum rule:

$$
\int_{0}^{\infty} \sigma_{1}(\omega) \mathrm{d} \omega=\pi n_{\mathrm{e}} e^{2} / 2 m_{\mathrm{e}}
$$

where $e$ is the elementary charge and $m_{\mathrm{e}}$ is the electron mass ${ }^{37,41}$. Therefore, the integration of a part of the spectral region $W$ between $E_{1}$ and $E_{2}$, given by:

$$
W=\int_{\mathrm{E}_{1}}^{\mathrm{E}_{2}} \sigma_{1}(E) \mathrm{d} E
$$

is proportional to the number of free charge carriers within that spectral region. By analyzing the change of $W$ with temperature we also gain a unique insight into the behaviour of the free charge carriers as the $\mathrm{MoS}_{2}$ sample is cooled.

The total spectral weight, $W_{\text {tot }}$, of the $\mathrm{MoS}_{2}$ across the measured spectral range of $0.6-45.0 \mathrm{eV}$ is shown in Fig. $3 \mathrm{~b}$ as a function of temperature. Upon cooling, $W_{\text {tot }}$ is nearly constant from 400 to $300 \mathrm{~K}$, increases generally from 300 to $150 \mathrm{~K}$, then decreases slowly below $150 \mathrm{~K}$ before a rapid drop-off below $100 \mathrm{~K}$. This indicates that there are more electrons with energies from 0.6 to $45.0 \mathrm{eV}$ between 300 and $150 \mathrm{~K}$ than at any other temperature, which means that above and below that temperature range, the number of electrons with energies outside of this spectral range is increasing. This is reversed below $150 \mathrm{~K}$ and the spectral weight decreases dramatically below $100 \mathrm{~K}$. The extra energy gained or lost in such a broad energy range comes from the potential energy of electron-electron correlations yielding strong Coulomb correlations below $100 \mathrm{~K}^{36}$. Interestingly, the soft $\mathrm{X}$-ray correlated-plasmons seen in Fig. 2 are observed to have different transition temperatures coinciding with the rise or fall in electron conductivity and thus electron density, as seen in Fig. 2 b. These shifts in spectral weight involve the loss or gain of a few tens of $\mathrm{eV}$ and thus cannot be explained by thermal activation alone as the energies associated with temperatures below $500 \mathrm{~K}$ are too small $(<43 \mathrm{meV})$. Furthermore, they also cannot be explained by electron-phonon interactions as in $\mathrm{MoS}_{2}$, the energy gained by these interactions is only $9 \mathrm{~cm}^{-1}$ or $1.1 \mathrm{meV}^{47}$. Similarly, exciton-plasmon interactions can also be discounted as the coupling strength is only $53 \mathrm{meV}^{48}$.

We now discuss the connection between the soft X-ray correlated-plasmons and spectral weight of each region $\left(W_{\mathrm{I}}, W_{\mathrm{II}}\right.$, and $W_{\text {III }}$ for regions I, II, and III, respectively). Figure $3 \mathrm{c}$ shows the relative change in $W$ of each of the three spectral regions from Fig. $3 \mathrm{a}$ as the sample is cooled from 400 to $40 \mathrm{~K}$. As temperature decreases from 400 to $300 \mathrm{~K}, W_{\text {III }}$ is nearly constant, while $W_{\mathrm{I}}$ and $W_{\text {II }}$ show only small changes. At this temperature range, we observe the soft X-ray correlated-plasmon at $35 \mathrm{eV}$ and its intensity is nearly constant. As the temperature further decreases from 300 to $250 \mathrm{~K}$, there is a sharp increase in all regions followed by a small and gradual decrease from 250 to $150 \mathrm{~K}$. The sharp increase between 300 and $250 \mathrm{~K}$ is the result of an increased number of electrons with energies that occupy these spectral ranges. As this occurs across all of the spectral regions, this reveals that the extra electrons are from above $45 \mathrm{eV}$. This means that we are seeing a drastic loss in electron energy of the order of several tens of $\mathrm{eV}$, which is far too large to be accounted for by thermal changes, or by electron-phonon or electron-plasmon interactions as stated earlier ${ }^{47,48}$. A loss of energy this large comes from the loss of electrons from the $35 \mathrm{eV}$ soft X-ray correlatedplasmon, resulting in the reduction of its intensity seen in Fig. 2a. This is accompanied with steadily enhancing, sharpening, and blue-shifting of low energy excitons (Fig. 1b-d and see also Supplementary Fig. 5).

The spectral weight of the low-energy excitons represented in $W_{\mathrm{I}}$ increases from 150 to $100 \mathrm{~K}$ (see also Fig. 3a). Conversely, $W_{\text {III }}$ decreases upon cooling whereas $\mathrm{W}_{\mathrm{II}}$ remains unaffected. We are seeing a transfer of spectral weight from region III to region I, whereby electrons from the high-energy bands of region III lose energy and contribute to the enhancement of the low-energy excitons. The fact that these low-energy excitons depend on such high-energy bands is a surprising result. The loss of spectral weight in region III upon cooling down to $100 \mathrm{~K}$ is also a result of an increase in electron energy due to the increasing number of electron-electron correlations. As the $\mathrm{MoS}_{2}$ cools, the electronic screening steadily reduces, giving rise to more electron-electron correlations across the material. The excitonic features A-D blueshift and sharpen with cooling, despite the fact that there is a loss in spectral weight from the higher energy regions to region 1 . This means that there are more electrons with energies between 0.6 and $3.4 \mathrm{eV}$ at lower temperatures than there were at higher temperatures and results in the magnitude of the excitonic peaks increasing. The blue-shifting of the peaks (Supplementary Fig. 5) is a result of the increasing potential energy due to the reduced electronic screening and steadily increasing electron-electron correlations ${ }^{49}$.

Further cooling below $100 \mathrm{~K}$, results in the drastic reduction of spectral weight in all regions are due to the formation of the soft $\mathrm{X}$-ray correlated-plasmons at $\sim 15$ and $\sim 30 \mathrm{eV}$. The loss in spectral weight at lower temperatures from the $W_{\text {II }}$ and $W_{\text {III }}$ regions represents a significant shift in electron density from this spectral range to even higher energies (above $45.0 \mathrm{eV}$ ) and to low energies (below $3.4 \mathrm{eV}$ ). The increase in electron energy of the order of 10 's of eV comes from long-range, high-energy electron-electron correlations, which are now prominent due to weakening electronic screening upon cooling. These electron-electron correlations form the soft X-ray correlated-plasmons at $\sim 15$ and $\sim 30 \mathrm{eV}$ in Fig. 2. It is clear that the massive spectral weight transfer over a broad energy range, which is a fingerprint of strong electronic correlations, is caused by the formation and reduction of the soft $\mathrm{X}$-ray correlated-plasmons at 15, 30, and $35 \mathrm{eV}$. Below $100 \mathrm{~K}$, the spectral weight of regions II and III remain relatively constant whilst region I shows an increase due to the continuing enhancement of the low-energy excitons down to $40 \mathrm{~K}$.

It is worth mentioning that the values of $n$ near the energy of the plasmons are nearly zero but remain positive, revealing that these are unconventional correlated-plasmons in the soft X-ray region (see also Supplementary Fig. 6). Note that correlated-plasmons in the near-IR and optical regions have previously been found within low- 

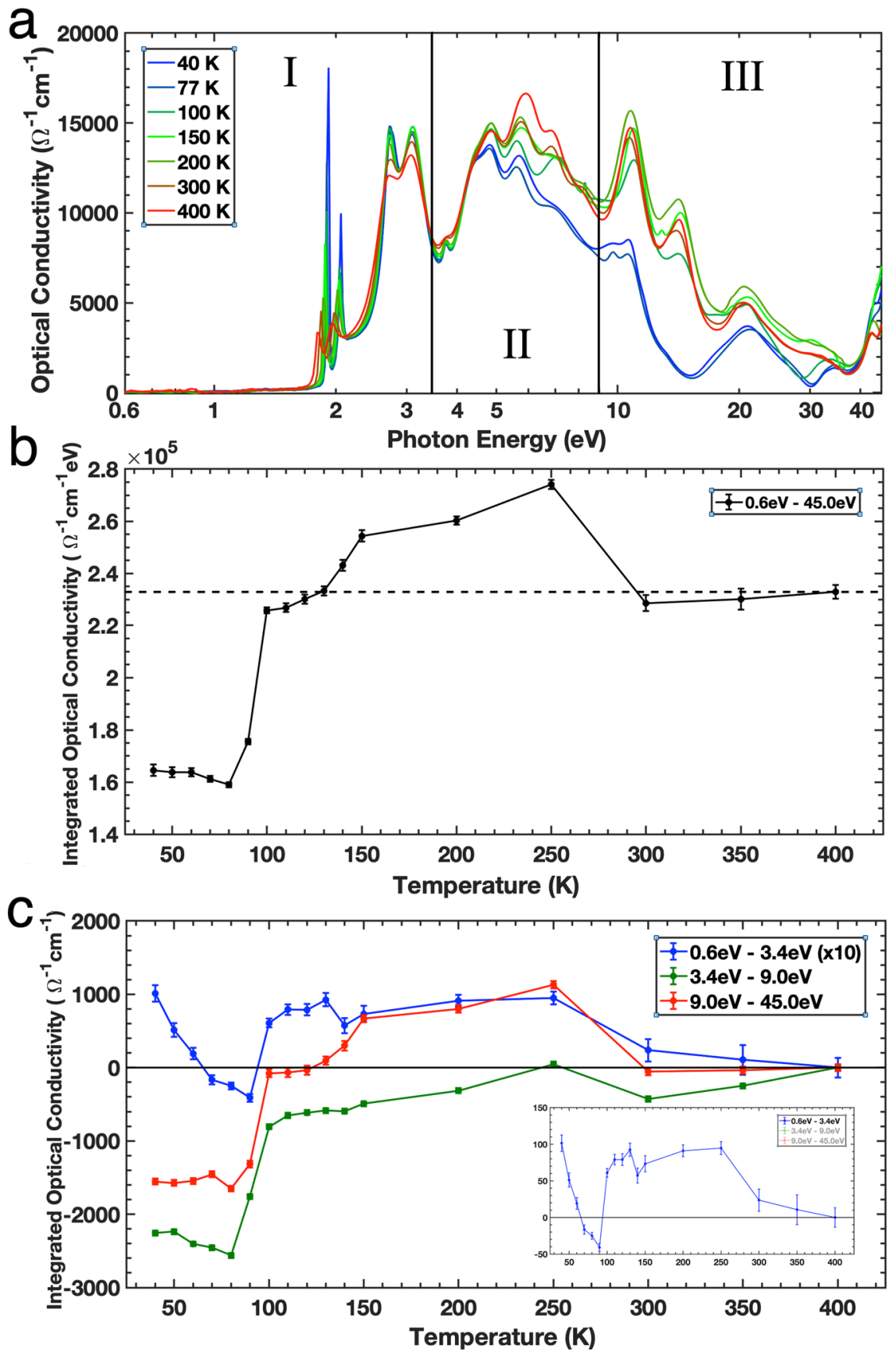

Fig. 3 Spectral weight transfer of $\mathbf{M o S}_{\mathbf{2}}$. a The optical conductivity of $\mathrm{MoS}_{2}$ of selected temperatures divided into three spectral regions. $\mathbf{b}$ The optical conductivity integrated over the whole spectrum as a function of temperature. Error bars are calculated using a 20 meV shift of spectral divide. c The relative change in integrated optical conductivity of the three different spectral regions as the sample is cooled from 400 to $40 \mathrm{~K}$. Region I has been magnified for clarity. Inset: Original values of Region I. Error bars are calculated using a $20 \mathrm{meV}$ shift of spectral divide.

dimensional Niobates further support that unconventional soft $\mathrm{X}$-ray correlated-plasmons in $\mathrm{MoS}_{2}$ are due to strong electronic correlations ${ }^{50}$. The corresponding values of $k$ are low, e.g. $k \sim 0.2$ at $30 \mathrm{eV}$, revealing low-loss correlated plasmons. This is very unusual because such high energies typically contained a considerable number of inter-band transitions, therefore one might expect to find a reasonably high absorption. The soft X-ray correlated-plasmons can occur in materials with a low free-charge density and arise from the collective oscillation of quasi-local (or correlated) electrons, which means they can have more than one plasmon energy. Correlated-plasmons are a new type of plasmon driven by electronic correlations and framing the unconventional correlated-plasmons as collective electron gas effects would not be accurate 50,51 . For the case of $\mathrm{MoS}_{2}$, by assuming a free electron gas, the equation for plasma frequency applies:

$$
\omega_{\mathrm{p}}=E_{\mathrm{p}} / \hbar=\sqrt{\frac{n e^{2}}{m_{\mathrm{e}} \varepsilon_{0}}},
$$

where $n$ is the electron density, $m_{\mathrm{e}}$ is the electron mass, $\varepsilon_{0}$ is the permittivity of free space, $e$ is the elementary charge, $\hbar$ is the reduced Planck's constant, and $\omega_{\mathrm{p}}$ and $E_{\mathrm{p}}$ are the plasmon frequency and energy respectively. The $15 \mathrm{eV}$ plasmon would, in conventional terms, have an electron density of $1.632 \times 10^{23} \mathrm{~cm}^{-1}$. 
A Out-of-plane $p$ - $d$ hybridizations $(z)$
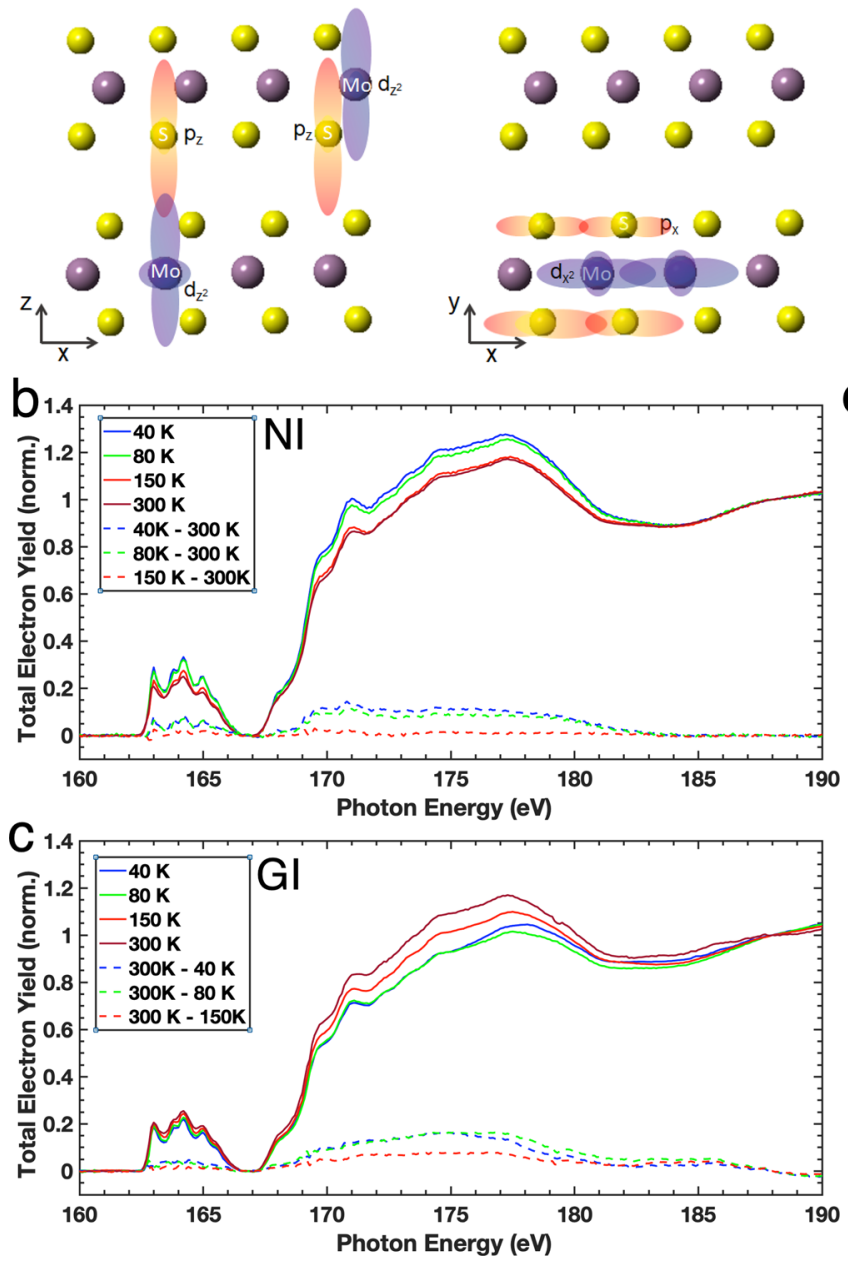

In plane $p$ - $d$ hybridizations (xy)
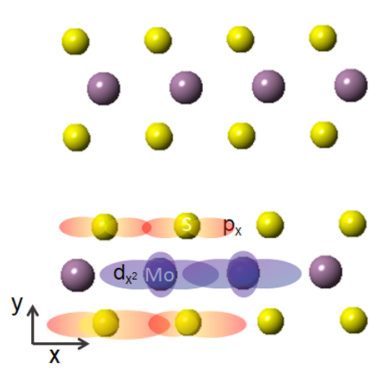

\section{e}
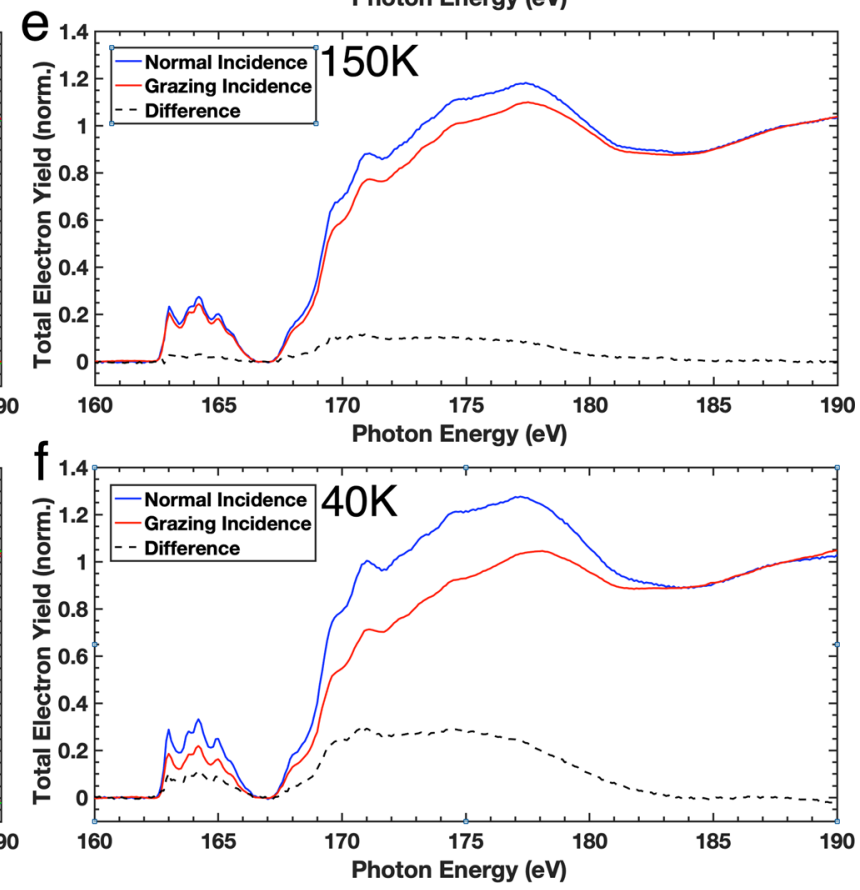

Fig. 4 Temperature-dependent X-ray absorption spectroscopy (XAS) spectra at $S L_{2,3}$ edges and their differences with respect to $300 \mathrm{~K}$ data on

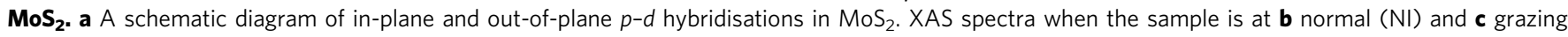
incidence (GI) angles, respectively, as a function of temperature. d-f Comparison of normal and grazing incidence XAS spectra for $300 \mathrm{~K}$, $150 \mathrm{~K}$, and $40 \mathrm{~K}$, respectively.

$\mathrm{MoS}_{2}$ has a mass density of $5.06 \mathrm{~g} / \mathrm{cm}$ and a molar mass of $160.07 \mathrm{~g} / \mathrm{mol}$, so assuming a perfect crystal, the molecular density is $1.904 \times 10^{22} \mathrm{~cm}^{-1}$. This would mean that about 8.6 free electrons per molecule would be needed to generate the $15 \mathrm{eV}$ plasmon. For the $30 \mathrm{eV}$ plasmon this would be 34.3 free electrons per molecule and the $35 \mathrm{eV}$ plasmon would need 46.7 free electrons per molecule. This is clearly not feasible and further supports that these soft X-ray correlated-plasmons are quantum oscillations of correlated-electrons.

To further reveal the origin of the soft X-ray correlated-plasmons and its transitions, we perform temperature-dependent soft X-ray absorption spectroscopy (XAS) at $S L_{2,3}$ edges of $\mathrm{MoS}_{2}$ as shown in Fig. 4. The XAS measurements are carried out using linear polarizations under two different geometries: normal incidence (NI), in which the photon polarization was parallel with the in-plane direction, and grazing incidence (GI), in which the photon polarization was in the out-of-plane direction. In-plane and out-of-plane polarization dependent XAS are common techniques to study inter-layer and intra-layer electronic coupling and dimensionality, and has been used extensively, especially in strongly correlated electron systems such as $\mathrm{La}_{2-\mathrm{x}} \mathrm{Ba}_{\mathrm{x}} \mathrm{CuO}_{4}$ and $\mathrm{Sr}_{14} \mathrm{Cu}_{24} \mathrm{O}_{41} 52,53$, which show strong polarisation dependence along the $\mathbf{a}, \mathbf{b}$, and $\mathbf{c}$ directions. Polarisation-dependent XAS reveals the dimensionality of the electronic structure of such materials. The strengthening of in-plane (intra-layer) electronic coupling and weakening of out-of-plane (inter-layer) electronic coupling are a clear indication that the system is electronically becoming more two-dimensional-like. The XAS data are normalized off-resonance, below and above the edges. The $S L_{3,2^{-}}$ edge consists of two main spectral features: the pre-edge spectra within $162-167 \mathrm{eV}$ corresponding to the transition from $\mathrm{S} 2 p_{\mathrm{j}=3 / 2,1 / 2}$ to S $3 p$-Mo $4 d$ hybridized states, and the larger postedge spectral features around $167-185 \mathrm{eV}$, which are attributed to the S $2 p_{3 / 2,1 / 2} \rightarrow \mathrm{S} 3 d$ (hybridized with Mo $5 p$ states) transition ${ }^{43}$. These $p-d$ hybridizations are illustrated in Fig. 4 a for both the inplane and out-of-plane directions within the layered $\mathrm{MoS}_{2}$. We note that the out-of-plane $p-d$ hybridizations consist of interlayer and intralayer correlations, while the in-plane $p-d$ hybridizations mostly consist of intralayer correlations.

In Fig. 4b, the temperature dependent XAS at NI, which probes the in-plane $p-d$ hybridizations, is shown. Upon cooling, the spectral weight increases below $150 \mathrm{~K}$, which reveals that the $p-d$ hybridizations within the layer become enhanced as the $\mathrm{MoS}_{2}$ is cooled. In contrast, this is not the case for the temperature dependent XAS at GI as shown in Fig. 4c. This probes the out-ofplane $p-d$ hybridizations and, upon cooling, the spectral weight decreases below $150 \mathrm{~K}$. This shows that as the temperature decreases, the $p-d$ hybridizations of interlayer and intralayers 
decrease in the $\mathbf{z}$-direction, resulting in a two-dimensional-like system at low temperatures.

Next, we compare the polarization dependent XAS taken at different temperatures (Fig. 4d-f) under these two different geometries. At $300 \mathrm{~K}$ (Fig. 4d), the XAS spectra for NI and GI are similar, revealing that the in-plane and out-of-plane $p-d$ hybridizations are isotropic at higher temperatures. This isotropic nature is a surprising result, revealing strong interlayer and intralayer correlations along the $\mathbf{z}$-direction, which are as strong as the intralayer correlations in the $\mathbf{x y}$-plane (Fig. 4a), yielding a three-dimensional-like system at high temperature and thus the soft X-ray correlated-plasmon has three-dimensional-like character. Upon cooling to $150 \mathrm{~K}$ and then $40 \mathrm{~K}$ (Fig. $4 \mathrm{e}$, f, respectively), there is a significant change in the absorption intensity between the NI and GI spectra. The $p-d$ hybridizations are now highly anisotropic in which the intralayer correlation in the $\mathbf{x y}$ plane (or in-plane $p-d$ hybridizations) are stronger than the interlayer and intralayer correlations in the $\mathbf{z}$-direction (or out-ofplane $p-d$ hybridizations), yielding a two-dimensional-like system. The anisotropy of the $p-d$ hybridizations weakens (enhances) the electronic screening within (across) the layers, respectively and leads to the suppression of the threedimensional-like soft X-ray correlated-plasmon at $\sim 35 \mathrm{eV}$ and the formation of the soft X-ray correlated-plasmons at $\sim 15$ and $\sim 30 \mathrm{eV}$, which instead, have a two-dimensional electronic character below $150 \mathrm{~K}$. We note that while the interlayer and intralayer atomic coupling gets stronger upon cooling, the electronic correlations behave differently depending on the hybridisations of the electron orbitals of the material ${ }^{47}$. This is because the degree of $p-d$ hybridizations are determined through bond angle and length between and within the S-Mo-S layers. As Fig. 4a shows, hybridizations get stronger because the angle between atoms is approaching $180^{\circ}$ (or away from $90^{\circ}$ ). We note that Raman spectroscopy shows significant blue-shifting with decreasing temperature, meaning that atoms within layers become closer and have stronger phonon coupling ${ }^{47}$, which then further enhances the out-of-plane $p-d$ hybridizations.

Further cooling below $100 \mathrm{~K}$, Coulomb correlations get stronger as well as the intralayer correlation enhancing the soft X-ray correlated-plasmons, as revealed from spectral-weight transfer (Fig. 3c). The transitions from the three-dimensional-like to the two-dimensional-like system which yield the unconventional soft $\mathrm{X}$-ray correlated-plasmons are quantum confinement effects in bulk-like $\mathrm{MoS}_{2}$. The high-energy bands, interlayer and intralayer correlations, and unconventional soft X-ray correlated-plasmons determine low-energy properties such as excitons.

To further reveal the roles of electron-electron and electron-hole interactions and high-energy bands in $\mathrm{MoS}_{2}$, Fig. $5 \mathrm{a}, \mathrm{b}$ show $\varepsilon_{1}$ and $\varepsilon_{2}$ calculations in a broad energy range, respectively. To understand the impact of higher energy bands, the number of valence bands used for these calculations is $N_{\mathrm{v}}=4$, while the number of conduction bands used are varied, $N_{\mathrm{c}}=10$ (blue lines) and $N_{\mathrm{c}}=16$ (green lines). Clearly, an increased number of high-energy conduction bands results in more interband transitions yielding an increase in calculated $\varepsilon_{2}$ at higher energies (Fig. 5b), which are qualitatively consistent with the experimental data (c.f. Fig. 1c). Interestingly, when the electron-hole interaction is turned on, the calculated $\varepsilon_{2}$ at high energies reduces dramatically and the spectral weight at high energies push toward low energies (Fig. 5c). This is qualitatively consistent with the low temperature data $(77 \mathrm{~K})$ in which spectral weight transfer occurs from high to low energies upon cooling as discussed earlier, with the effects being the enhancement of the excitons, as seen in the low energy part of the calculated $\varepsilon_{2}$ (Fig. 5c). Furthermore, $\varepsilon_{2}$ between $12-13 \mathrm{eV}$ does show significant reduction as low as $\sim 2-3$ and $\varepsilon_{1}$ is near zero (as seen in
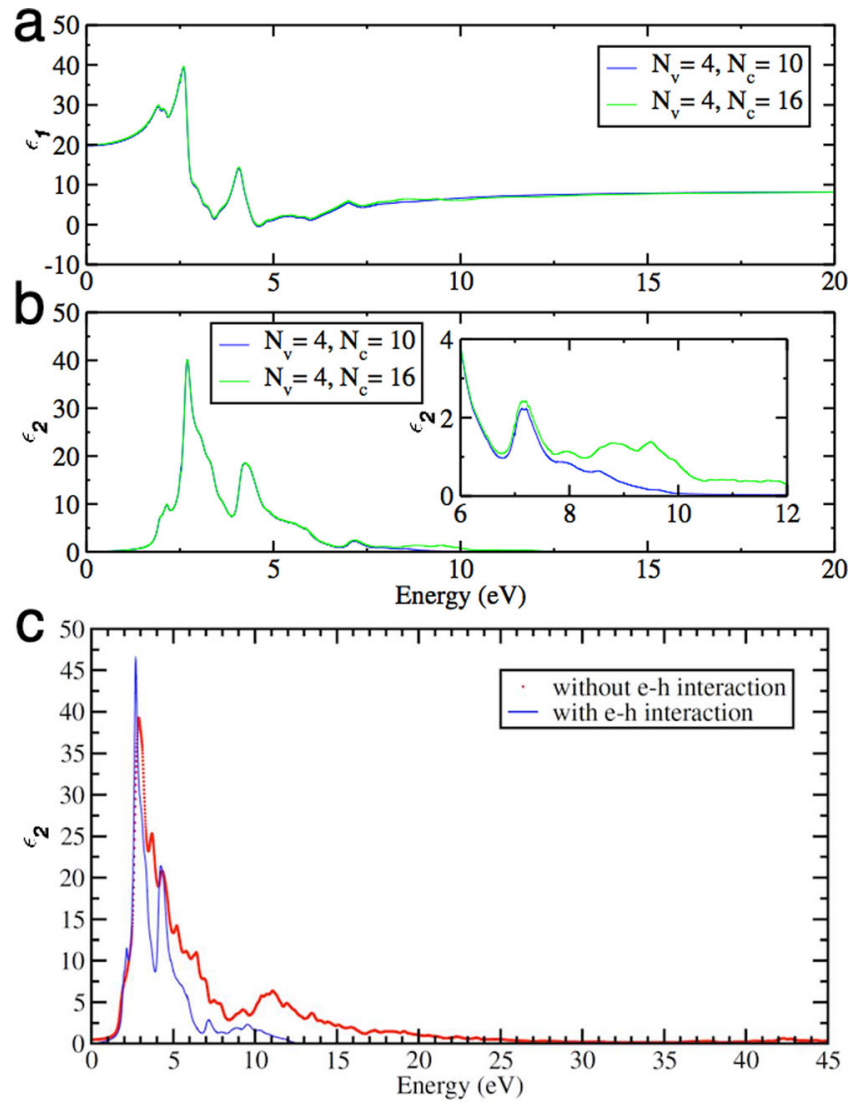

Fig. 5 High-energy dielectric function calculations. The $\mathbf{a}$ real and $\mathbf{b}$ imaginary parts of the complex dielectric function calculated with four valence bands and 10 (blue) and 16 (green) conduction bands. c Calculations of the imaginary part of the complex dielectric function with and without electron-hole interactions.

Supplementary Fig. 2c). For further support, the roles of number of valance and conductions bands to the low energy excitations are sown in Supplementary Fig. 7. By combining our experimental results and theoretical calculations, we find that electron-electron and electron-hole interactions, and highenergy bands are important to comprehensively determine the electronic structure and optical properties over a broad energy range. The strength of these interactions, and thus the electronic screening, changes as a function of temperature and the highenergy bands play important role in determining the low-energy excitations, including low-energy excitons in $\mathrm{MoS}_{2}$.

This also succinctly explains the behaviour of the FWHM of the low-energy A and B excitons seen in Fig. 1d. When the pair of soft X-ray correlated-plasmons at 15 and $30 \mathrm{eV}$ occurs around $100 \mathrm{~K}$ (due to electron-electron correlations and hybridizations), the low-energy excitons are directly affected, as evidenced by the increase and subsequent decrease of the exciton's FWHM, and thus their lifetime. Similarly, the changes that occur in exciton A from 350 to $250 \mathrm{~K}$ are due to the reduction of the 3D-like soft $\mathrm{X}$-ray correlated-plasmons below $350 \mathrm{~K}$. This is very unusual because the energy separations between the soft X-ray correlatedplasmons are as high as $\sim 28 \mathrm{eV}$. This reveals a strong coupling between the high-energy and low-energy bands as shown through the coupling of the correlated-plasmons and low-energy excitons.

In summary, the unusual $3 \mathrm{D}$ character of the electronic structure at room temperature and $3 \mathrm{D}$ to $2 \mathrm{D}$-like electronic transitions observed upon cooling in $\mathrm{MoS}_{2}$ reveal the importance of the interplay of electronic correlations and $p-d$ hybridizations between different S-Mo-S layers (interlayer correlations) and 
within the S-Mo-S layers (intralayer correlations), which are determined by the length and bonding angle degrees of freedom. Such an interplay yields many-body soft X-ray correlated-plasmons with low-loss, which also opens up the applications on the use of transition metal dichalcogenides into plasmonic nanolithography within a broad energy range, from the deep ultraviolet to the soft X-ray. The advantages are that the soft X-ray correlated-plasmons exists even well above room temperature and occur naturally in the bulk form, therefore it simplifies the fabrication and integration processes of plasmonic nanolithography devices ${ }^{54}$. Furthermore, as a function of temperature, one can select different types of correlated-plasmons. Our result shows the importance of strong electronic, interlayer and intralayer correlations in determining the complete optical and electronic structures of strongly-correlated transition metal dichalcogenides.

\section{Methods}

Sample preparation. The bulk, single crystal $\mathrm{MoS}_{2}$ was purchased from HQ Graphene and a single flake with a flat surface was used in both the spectroscopic ellipsometry and reflectivity measurements. In both experiments the sample is heated to high temperatures and left for several hours to remove surface contaminants before any measurements were taken. Characterizations of the crystal can be found in http://www.hqgraphene.com/MoS2.php, which includes X-ray diffraction (XRD), energy dispersive X-ray spectroscopy (EDX), and Raman spectroscopy.

Muller-matrix spectroscopic ellipsometry. Muller-matrix spectroscopic ellipsometry measurements are carried out using a variable-angle spectroscopic ellipsometer (V-VASE, J.A. Woollam Co.) with a rotating analyser and compensator ${ }^{37}$. Two sets of measurements on bulk $\mathrm{MoS}_{2}$ are taken in the energy range of $0.6-5.5 \mathrm{eV}$ whilst the samples are inside an ultra-high vacuum cryostat with a base pressure of $10^{-8} \mathrm{mbar}$. The sample is cooled to $77 \mathrm{~K}$ using liquid Nitrogen and measurements are taken at angles of 68,70 , and 72 degrees, which are limited in range by the UHV windows. The sample is then heated slowly to temperatures of $100,150,200,250,300,350$, and $400 \mathrm{~K}$. Each of these measurements are taken at a single angle of $70^{\circ}$ for each temperature.

The second set of measurements involve the $\mathrm{MoS}_{2}$ being cooled to $40 \mathrm{~K}$ using liquid Helium and then heated slowly to temperatures of 40, 50, 60, 70, 80, 90, 100, $110,120,130,140$, and $150 \mathrm{~K}$. The cooling/heating of the samples is uniform and slow and they are kept at each temperature for $2 \mathrm{~h}$ as the measurements are taken. The Muller-matrix spectroscopic ellipsometry directly measures change of amplitude $\Psi$ and phase $\Delta$ and from which we obtain the complex dielectric function, loss function, and reflectivity.

Soft X-ray reflectance measurements. The reflectance measurements are taken at the Soft X-ray-Ultraviolet (SUV) beamline equipped with a unique ultra-high vacuum (UHV) diffractometer of 5-circle geometry, full control of polarization and low temperature capabilities at the Singapore Synchrotron Light Source (SSLS) ${ }^{39}$. The single crystal $\mathrm{MoS}_{2}$ is held in a vacuum chamber at $10^{-9}$ mbar and illuminated with a high-intensity photon beam from the synchrotron radiation. The energy of the photon beam is then tuned from 3.5 to $45 \mathrm{eV}$ and the reflected light is measured by a diode positioned at an angle of $20^{\circ}$ with respect to the surface. The data is then normalised by the recorded current of the beam to remove the effects of beam fluctuation during the recording of the data and then also normalised with the throughput of the beam to get the total reflectance of the material.

The temperature of the $\mathrm{MoS}_{2}$ was increased from 77 to $400 \mathrm{~K}$ via control of the sample holder temperature with a temperature gauge on the sample. The sample is heated slowly to temperatures of $100,150,200,250,300,350$, and $400 \mathrm{~K}$. The second set of measurements involve the $\mathrm{MoS}_{2}$ sample being cooled to $40 \mathrm{~K}$ using liquid Helium and then heated slowly to temperatures of 40,50, 60, 70, 80, 90, 100, $110,120,130,140$, and $150 \mathrm{~K}$. The heating of the sample is uniform and slow and they are kept at each temperature for $30 \mathrm{~min}$ before the measurements are taken and for $1 \mathrm{~h}$ during the measurements.

\section{Soft X-ray absorption spectroscopy. The temperature-dependent and} polarization-dependent soft X-ray absorption spectroscopy (XAS) measurements are carried out at the SUV beamline equipped with a unique ultra-high vacuum (UHV) diffractometer of 5-circle geometry, full control of polarization and low temperature capabilities of SSLS ${ }^{39}$. In particular, the $S L_{3,2}$-edge spectra are collected in the total electron yield (TEY) mode in the energy range of $155-195 \mathrm{eV}$. The $\mathrm{MoS}_{2}$ is held in a vacuum chamber at $10^{-9} \mathrm{mbar}$ and illuminated with a high-intensity photon beam from the synchrotron radiation. The sample is subjected to linearly polarized X-ray photons under both normal and grazing incidences providing in-plane and out-ofplane polarizations, respectively. The X-ray photons are then absorbed by the sample and produces electric current, which is counted by a very sensitive current meter. The data are normalized to the incident $\mathrm{x}$-ray beam intensity, then an energy-independent background is subtracted and scaled to get the total electron yield of the sample. The temperature of the $\mathrm{MoS}_{2}$ is increased from 40 to $300 \mathrm{~K}$ via control of the sample holder temperature with a temperature gauge on the sample. The sample is heated slowly to temperatures of 80,150 , and $300 \mathrm{~K}$.

Band-structure calculations. The ground state structure, the Kohn-Sham states and the corresponding energies are calculated using density functional theory as implemented in the Quantum ESPRESSO package ${ }^{55}$. A cut-off energy of $40 \mathrm{Ry}$ is used for the wave-function expansion. We employ a fully relativistic pseudopotential to account for core electrons and further to calculate the spin-orbit coupling. The Brillouin zone is sampled using $12 \times 12 \times 1 \mathbf{k}$-point grid.

The many-body effects are calculated using many-body perturbation theory implemented in the Yambo code. The many-body corrections to the previously calculated Kohn-Sham energies are calculated within $\mathrm{G}_{0} \mathrm{~W}_{0}$ approximation to the self-energy. The absorption spectrum, i.e., the complex dielectric function, are then calculated by solving the Bethe-Salpeter equation. As for the calculation of the Bethe-Salpeter matrix, we use the same sample of the Brillouin zone interpolated to larger grid by using double-grid technique. We employ 200 unoccupied bands to build the polarizability.

The size of the matrix that has to be diagonalized to solve Bethe-Salpeter equation is $2 \times N_{\mathrm{v}} \times N_{\mathrm{c}} \times N_{\mathrm{k}}$, where 2 comes from the spin degree of freedom, $N_{\mathrm{v}}$ and $N_{\mathrm{c}}$ are the number of valence and conduction bands, and $N_{\mathrm{k}}$ is the number of k-points in the Brillouin zone. With the symmetry of the system using a doublegrid method, we have only 78 inequivalent $\mathbf{k}$-points, yet the size of the Bethe-Salpeter matrix can easily reach thousands. The number of valence and conduction bands can be chosen to be small if one is only interested in the visible light region. Since we are interested in calculating the spectrum up to $20 \mathrm{eV}$, here we use $N_{\mathrm{v}}=4$ and $N_{\mathrm{c}}=10$ and 16 .

\section{Data availability}

All data that supports the findings of this study are available from the corresponding author upon reasonable request.

Received: 2 September 2020; Accepted: 27 October 2021; Published online: 30 November 2021

\section{References}

1. Mott, N. F. The basis of the electron theory of metals, with special reference to the transition metals. Proc. Phys. Soc. Sect. A 62, 416 (1949).

2. Hubbard, J. Electron correlations in narrow energy bands. II. The degenerate band case. Proc. R. Soc. Ser. A Math. Phys. Sci. 277, 237 (1964).

3. Basov, D. N., Averitt, R. D., Van Der Marel, D., Dressel, M. \& Haule, K. Electrodynamics of correlated electron materials. Rev. Mod. Phys. 83, 471 (2011).

4. Jonker, G. H. \& Van Santen, J. H. Ferromagnetic compounds of manganese with perovskite structure. Physica 16, 337 (1950).

5. Jin, S. et al. Thousandfold change in resistivity in magnetoresistive La-Ca-MnO films. Science 264, 413-415 (1994).

6. Imada, M., Fujimori, A. \& Tokura, Y. Metal-insulator transition. Rev. Mod. Phys. 70, 1039 (1998).

7. Radisavljevic, B. \& Kis, A. Mobility engineering and a metal-insulator transition in monolayer $\mathrm{MoS}_{2}$. Nat. Mater. 12, 815 (2013).

8. Santoso, I. et al. Observation of room-temperature high-energy resonant excitonic effects in graphene. Phys. Rev. B 84, 081403 (2011).

9. Yong, Z. et al. Emerging giant resonant exciton induced by Ta substitution in anatase $\mathrm{TiO}_{2}$ : a tunable correlation effect. Phys. Rev. B 93, 205118 (2016).

10. Das, P. K. et al. Electronic correlation determining correlated plasmons in $\mathrm{Sb}$ doped $\mathrm{Bi}_{2} \mathrm{Se}_{3}$. Phys. Rev. B 100, 115109 (2019).

11. Whitcher, T. J. et al. Correlated plasmons in the topological insulator $\mathrm{Bi}_{2} \mathrm{Se}_{3}$ induced by long-range electron correlations. NPG Asia Mater. 12, 1 (2020).

12. Hubbard, J. Electron correlations in narrow energy bands. Proc. R. Soc. Ser. A 276, 238 (1963)

13. Eskes, H., Meinders, M. B. J. \& Sawatzky, G. A. Anomalous transfer of spectral weight in doped strongly correlated systems. Phys. Rev. Lett. 67, 1035 (1991).

14. Ohta, Y., Tsutsui, K., Koshibae, W., Shimozato, T. \& Maekawa, S. Evolution of the in-gap state in high- $\mathrm{T}_{\mathrm{c}}$ cuprates. Phys. Rev. B 46, 14022 (1992).

15. Dagotto, E. Correlated electrons in high-temperature superconductors. Rev. Mod. Phys. 66, 763 (1994).

16. Novoselov, K. S. et al. Two-dimensional atomic crystals. Proc. Natl Acad. Sci. 102, 10451 (2005)

17. Mak, K. F., Lee, C., Hone, J., Shan, J. \& Heinz, T. F. Atomically thin $\mathrm{MoS}_{2}$ : a new direct-gap semiconductor. Phys. Rev. Lett. 105, 136805 (2010).

18. Wang, Q. H., Kalantar-Zadeh, K., Kis, A., Coleman, J. N. \& Strano, M. S. Electronics and optoelectronics of two-dimensional transition metal dichalcogenides. Nat. Nanotechnol. 7, 699-712 (2012). 
19. Komsa, H. \& Krasheninnikov, A. Effects of confinement and environment on the electronic structure and exciton binding energy of $\mathrm{MoS}_{2}$ from first principles. Phys. Rev. B 86, 241201 (2012).

20. Qiu, D. Y., Jornada, F. H. \& Louie, S. G. Optical spectrum of $\mathrm{MoS}_{2}$ : manybody effects and diversity of exciton states. Phys. Rev. Lett. 111, 216805 (2013).

21. Ridolfi, E., Lewenkopf, C. H. \& Pereira, V. M. Excitonic structure of the optical conductivity in $\mathrm{MoS}_{2}$ monolayers. Phys. Rev. B 97, 205409 (2018).

22. Splendiani, A. et al. Emerging photoluminescence in monolayer $\mathrm{MoS}_{2}$. Nano Lett. 10, 1271-1275 (2010)

23. Tonndorf, P. et al. Photoluminescence emission and Raman response of monolayer $\mathrm{MoS}_{2}, \mathrm{MoSe}_{2}$, and $\mathrm{WSe}_{2}$. Opt. Express 21, 4908-4916 (2013).

24. Mak, K. F., He, K., Shan, J. \& Heinz, T. F. Control of valley polarization in monolayer $\mathrm{MoS}_{2}$ by optical helicity. Nat. Nanotechnol. 7, 494-498 (2012).

25. Xiao, D., Liu, G.-B., Feng, W., Xu, X. \& Yao, W. Coupled spin and valley physics in monolayers of $\mathrm{MoS}_{2}$ and other group-VI dichalcogenides. Phys. Rev. Lett. 108, 196802 (2012).

26. Wilson, J. A. \& Yoffe, A. D. Transition metal dichalcogenides discussion and interpretation of observed optical, electrical and structural properties. Adv. Phys. 18, 193 (1969).

27. Bullett, D. W. Electronic band structure and bonding in transition metal layered dichalcogenides by atomic orbital methods. J. Phys. C11, 4501 (1978).

28. Coehoorn, R. et al. Electronic structure of $\mathrm{MoSe}_{2}, \mathrm{MoS}_{2}$, and $\mathrm{WSe}_{2}$. I. Bandstructure calculations and photoelectron spectroscopy. Phys. Rev. B 35, 6195 (1987).

29. Zhu, B., Zeng, H., Dai, J., Gong, Z. \& Cui, X. Anomalously robust valley polarization and valley coherence in bilayer WS2. Proc. Natl Acad. Sci. 111, 11606 (2014).

30. Reed, J. P. et al. The effective fine-structure constant of freestanding graphene measured in graphite. Science 330, 805-808 (2010).

31. Cao, Y. et al. Unconventional superconductivity in magic-angle graphene superlattices. Nature 556, 43 (2018).

32. Zeppenfeld, K. Electron energy losses and optical anisotropy of $\mathrm{MoS}_{2}$ single crystals. Opt. Commun. 1, 377 (1970).

33. Beal, A. R. \& Hughes, H. P. Kramers-Kronig analysis of the reflectivity spectra of $2 \mathrm{H}-\mathrm{MoS}_{2}, 2 \mathrm{H}-\mathrm{MoSe} 2$ and 2H-MoTe2. J. Phys. C12, 881 (1979).

34. Ohno, Y. Kramers-Kronig analysis of reflection electron-energy-loss spectra measured with a cylindrical mirror analyzer. Phys. Rev. B 39, 8209 (1989).

35. Fujiwara, H. Spectroscopic Ellipsometry: Principles and Applications. (Wiley, 2007).

36. Rusydi, A. et al. Metal-insulator transition in manganites: changes in optical conductivity up to $22 \mathrm{eV}$. Phys. Rev. B 78, 125110 (2008).

37. Asmara, T. C., Santoso, I. \& Rusydi, A. Self-consistent iteration procedure in analyzing reflectivity and spectroscopic ellipsometry data of multilayered materials and their interfaces. Rev. Sci. Instrum. 85, 3663 (2014).

38. Schmidt, D., You, L., Chi, X., Wang, J. \& Rusydi, A. Anisotropic optical properties of rhombohedral and tetragonal thin film $\mathrm{BiFeO}_{3}$ phases. Phys. Rev. $B$ 92, 075310 (2015).

39. Yu, X. J., Diao, C. Z., Venkatesan, T., Breese, M. B. H. \& Rusydi, A. A soft Xray-ultraviolet (SUV) beamline and diffractometer for resonant elastic scattering and ultraviolet-vacuum ultraviolet reflectance at the Singapore synchrotron light source. Rev. Sci. Instrum. 89, 113113 (2018).

40. Henke, B. L., Gullikson, E. M. \& Davis, J. C. X-ray interactions: photoabsorption, scattering, transmission, and reflection at $\mathrm{E}=50-30000 \mathrm{eV}$, $\mathrm{Z}=1$-92. At. Data Nucl. Data Tables 54, 181 (1993).

41. van der Marel, D. Strongly Correlated Systems 269-296 (Springer, 2015).

42. Peng, B. et al. Thermal conductivity of monolayer $\mathrm{MoS}_{2}, \mathrm{MoSe}_{2}$, and $\mathrm{WS}_{2}$ : interplay of mass effect, interatomic bonding and anharmonicity. RSC Adv. 6, 5767 (2016).

43. Parija, A. et al. Mapping catalytically relevant edge electronic states of $\mathrm{MoS}_{2}$. ACS Cent. Sci. 4, 493-503 (2018).

44. Nerl, H. C. et al. Probing the local nature of excitons and plasmons in fewlayer $\mathrm{MoS}_{2}$. NPJ 2D Mater. Appl. 1, 2 (2017).

45. Li, X. et al. Pressure and temperature-dependent Raman spectra of $\mathrm{MoS}_{2}$ film. Appl. Phys. Lett. 109, 242101 (2016).

46. Bandaru, N. et al. Effect of pressure and temperature on structural stability of $\mathrm{MoS}_{2}$. J. Phys. Chem. C 118, 3230 (2014).

47. Guo, X., Chen, H., Wen, X. \& Zheng, J. Electron-phonon interactions in $\mathrm{MoS}_{2}$ probed with ultrafast two-dimensional visible/far-infrared spectroscopy. J. Chem. Phys. 142, 212447 (2015).

48. Liu, W. et al. Strong exciton-plasmon coupling in $\mathrm{MoS}_{2}$ coupled with plasmonic lattice. Nano Lett. 16, 1262 (2016)

49. Whitcher, T. J. et al. Importance of electronic correlations and unusual excitonic effects in formamidinium lead halide perovskites. Phys. Rev. X 8, 021034 (2018).
50. Asmara, T. C. et al. Tunable and low-loss correlated plasmons in Mott-like insulating oxides. Nat. Commun. 8, 156271 (2017).

51. Chaudhuri, A. et al. Origin of quasilocal plasmons in Nb-substituted $\mathrm{EuTiO}_{3}$. Phys. Rev. B 100, 085145 (2019).

52. Abbamonte, P. et al. Spatially modulated 'mottness' in $\mathrm{La}_{2-\mathrm{x}} \mathrm{Ba}_{\mathrm{x}} \mathrm{CuO}_{4}$. Nat Phys. 1, 155 (2005).

53. Abbamonte, P. et al. Crystallization of charge holes in the spin ladder of $\mathrm{Sr} 14$ Cu 24 O 41. Nature 431, 1078 (2004).

54. Srituravanich, W. et al. Flying plasmonic lens in the near field for high-speed nanolithography. Nat. Nanotechnol. 3, 733-737 (2008).

55. Giannozzi, P. et al. QUANTUM ESPRESSO: a modular and open-source software project for quantum simulations of materials. J. Phys. Condens. Matt 21, 395502 (2009).

\section{Acknowledgements}

This work is supported by Singapore Ministry of Education (MOE) AcRF Tier-2 (MOE2017 T2-1-135, MOE2018-T2-2-117, and MOE2019-T2-1-163), MOE-AcRF Tier 1 (R-144-000-423-114, R-144-000-398-114, R-144-000-379-114 and R-144-000-368112), the Singapore National Research Foundation under its Competitive Research Funding (No. R-398-000-087-281) and under its Medium Sized Centre Program (Centre for Advanced 2D Materials and Graphene Research Centre), NUS YIA, the 2015 PHC Merlion Project and NUS Core Support C-380-003-003-001. The authors would like to acknowledge the Singapore Synchrotron Light Source (SSLS) for providing the facility necessary for conducting the research. The SSLS is a National Research Infrastructure under the National Research Foundation Singapore.

\section{Author contributions}

A.R. designed new experimental techniques and facilities of high-energy spectroscopic ellipsometry coupled with Mueller-matric Spectroscopic ellipsometry. T.J.W. and A.R. analyze high-energy spectroscopic ellipsometry coupled with Mueller-matric Spectroscopic ellipsometry data comprehensively. T.J.W., X.C. and A.R. performed spectroscopic ellipsometry measurements. A.D.F., D.C., T.J. and A.R. performed high-energy reflectivity and X-ray absorption measurements. A.S., M.A.M. and A.R. performed theoretical calculations. AR and TJW wrote the manuscript with inputs from A.D.F., D.C., X.C., A.S., T.C.A., M.B.H.B., A.H.C.N., A.T.S.W. and M.A.M. A.R. lead and supervised the project.

\section{Competing interests}

The authors declare no competing interests.

\section{Additional information}

Supplementary information The online version contains supplementary material available at https://doi.org/10.1038/s41467-021-27182-y.

Correspondence and requests for materials should be addressed to T. J. Whitcher or A. Rusydi.

Peer review information Nature Communications thanks Bin Chen, and the other, anonymous, reviewer for their contribution to the peer review of this work.

Reprints and permission information is available at http://www.nature.com/reprints

Publisher's note Springer Nature remains neutral with regard to jurisdictional claims in published maps and institutional affiliations.

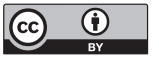

Open Access This article is licensed under a Creative Commons Attribution 4.0 International License, which permits use, sharing, adaptation, distribution and reproduction in any medium or format, as long as you give appropriate credit to the original author(s) and the source, provide a link to the Creative Commons license, and indicate if changes were made. The images or other third party material in this article are included in the article's Creative Commons license, unless indicated otherwise in a credit line to the material. If material is not included in the article's Creative Commons license and your intended use is not permitted by statutory regulation or exceeds the permitted use, you will need to obtain permission directly from the copyright holder. To view a copy of this license, visit http://creativecommons.org/ licenses/by/4.0/.

(C) The Author(s) 2021 